\title{
Norms of the Successive Overrelaxation Method
}

\author{
By David R. Kincaid*
}

\begin{abstract}
Norms, primarily of theoretical interest, of the successive overrelaxation method, with a relaxation parameter other than the optimum one, are studied. New results on this subject are presented and compared with those of Wachspress [8].
\end{abstract}

1. Introduction. A well-known iterative method for solving large systems of linear equations which arise from the discretization of elliptic partial differential equations is the successive overrelaxation method. Norms of this method with the optimum relaxation parameter have been studied by Sheldon [6], Golub and Varga [2], Young and Kincaid [9], and Young [11]. Until recently, the only study of norms of the successive overrelaxation method, with a relaxation parameter other than the optimum one, seems to be that by Wachspress [8]. New results on this subject are presented here and compared with those of Wachspress.

We consider solving the system of equations of the form $A x=b$, where

$$
A=\left(\begin{array}{ll}
D_{1} & H_{1} \\
H_{2} & D_{2}
\end{array}\right),
$$

such that $D_{1}$ and $D_{2}$ are square diagonal matrices. Here, $A \in \mathrm{R}^{N, N}$ is a real, $N \times N$ nonsingular positive definite** matrix with nonzero diagonal elements, $b \in \mathbf{R}^{N}$ is a given vector, and $x$ is the solution vector which is to be determined. Letting $D=\operatorname{diag} A$, we note that

$$
B=I-D^{-1} A=\left(\begin{array}{ll}
0 & F \\
G & 0
\end{array}\right),
$$

where $F=-D_{1}^{-1} H_{1}$ and $G=-D_{2}^{-1} H_{2}$.

2. Successive Overrelaxation Method. The successive overrelaxation (SOR) method for solving (1.1) is given by

$$
\begin{aligned}
& x_{1}^{(m+1)}=\omega\left\{F x_{2}^{(m)}+g_{1}\right\}+(1-\omega) x_{1}^{(m)}, \\
& x_{2}^{(m+1)}=\omega\left\{G x_{1}^{(m+1)}+g_{2}\right\}+(1-\omega) x_{2}^{(m)},
\end{aligned}
$$

Received August 23, 1971.

AMS 1969 subject classifications. Primary 6535; Secondary 1501, 6566.

Key words and phrases. Successive overrelaxation method, SOR method, norms, $D^{1 / 2}$-norm, positive definite, $A^{1 / 2}$-norm, relaxation parameter.

* Work on this paper was supported in part by U.S. Army Research Office (Durham) Grant DA-ARO(D)-31-124-G1050 and National Science Foundation Grant GP-23655 with The University of Texas at Austin.

** A real matrix $A$ is positive definite if $A$ is a nonsingular symmetric matrix and if the inner product $(x, A x)>0$ for all $x \neq 0 \in \mathbf{R}^{N}$.

Copyright (C) 1972, American Mathematical Society 
where $\omega$ is the relaxation parameter such that $0<\omega<2$. The optimum relaxation parameter is $\omega_{b}=2 /\left(1+\left(1-\bar{\mu}^{2}\right)^{1 / 2}\right)$, where $\bar{\mu}=S(B)$, the spectral radius*** of $B$. The SOR iterative matrix is

$$
\begin{aligned}
\mathscr{L}_{\omega} & =(I-\omega L)^{-1}((1-\omega) I+\omega U) \\
& =\left(\begin{array}{cc}
I_{1} & 0 \\
-\omega G & I_{2}
\end{array}\right)^{-1}\left(\begin{array}{cc}
(1-\omega) I_{1} & \omega F \\
0 & (1-\omega) I_{2}
\end{array}\right) \\
& =\left(\begin{array}{cc}
(1-\omega) I_{1} & \omega F \\
(1-\omega) \omega G & \omega^{2} G F+(1-\omega) I_{2}
\end{array}\right),
\end{aligned}
$$

where $L$ and $U$ are strictly lower triangular and strictly upper triangular matrices such that $B=L+U$. After $m$ iterations of the SOR method, we obtain the following iterative matrix:

$$
\mathfrak{L}_{\omega}^{m}=\left(\begin{array}{cc}
(1-\omega) \alpha_{2 m-2}(G, F, \omega) & \alpha_{2 m-1}(F, G, \omega) \\
(1-\omega) \alpha_{2 m-1}(G, F, \omega) & \alpha_{2 m}(F, G, \omega)
\end{array}\right),
$$

where

$$
\begin{aligned}
\alpha_{0}(G, F, \omega) & =I_{1}, \quad \alpha_{0}(F, G, \omega)=I_{2}, \quad \alpha_{1}(X, Y, \omega)=\omega X, \\
\alpha_{k}(X, Y, \omega) & =\alpha_{k-1}(Y, X, \omega) \omega X+(1-\omega) \alpha_{k-2}(X, Y, \omega), \quad k \geqq 2 .
\end{aligned}
$$

3. Norms of the SOR Method. From Kincaid [4], we have the following general result for the $A_{\beta}^{1 / 2}$-norm of the SOR method where $A_{\beta}=D(I-\beta B) .^{\dagger}$

THEOREM 3.1. If $A$ is a positive definite matrix of the form (1.1), then, for $m \geqq 1$ and $0 \leqq \beta \leqq 1$,

$$
\begin{aligned}
\left\|\mathscr{L}_{\omega}^{m}\right\|_{A^{1 / 2}} \leqq \max _{-\bar{\mu} \leqslant \mu \leqslant \bar{\mu}}\left\{\gamma_{\beta}(m, \mu, \omega)+\left(\gamma_{\beta}^{2}(m, \mu, \omega)+(1-\omega)^{2 m}\right)^{1 / 2}\right\}, & \\
& 0<\omega<2,
\end{aligned}
$$

where

$$
\gamma_{\beta}(m, \mu, \omega)=\frac{1}{2}\left\{(2-\omega)^{2}+\frac{\omega^{2} \mu^{2}(1-\beta)^{2}}{1-\mu^{2} \beta^{2}}\right\}^{1 / 2}\left|\alpha_{2 m-1}(\mu, \omega)\right|
$$

and

$$
\begin{aligned}
& \alpha_{0}(\mu, \omega)=1, \quad \alpha_{1}(\mu, \omega)=\omega \mu, \\
& \alpha_{k}(\mu, \omega)=\omega \mu \alpha_{k-1}(\mu, \omega)+(1-\omega) \alpha_{k-2}(\mu, \omega), \quad k \geqq 2 .
\end{aligned}
$$

The relevant $2 \times 2$ matrix for determining (3.1) from (2.3) is the following (see Kincaid [5] for details):

*** The spectral radius of a matrix $S$ is denoted $S(S)$ and is the maximum of the moduli of the eigenvalues of $g$.

$\dagger$ By Kincaid [4], if $A$ is positive definite, then $A_{\beta}=D(I-\beta B)$ is positive definite for $0 \leqq \beta \leqq 1$.

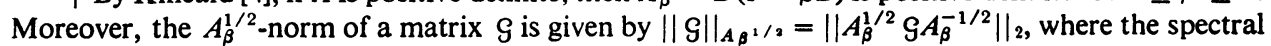
norm of a matrix $\mathfrak{F C}$ is given by $\| \mathcal{F C}_{2}^{2}=S\left(\mathcal{F C}^{H}{ }^{H}\right)$. 


$$
M_{m}(\mu, \omega)=\left(\begin{array}{cc}
(1-\omega) \alpha_{2 m-2}(\mu, \omega) & \alpha_{2 m-1}(\mu, \omega) \\
(1-\omega) \alpha_{2 m-1}(\mu, \omega) & \alpha_{2 m}(\mu, \omega)
\end{array}\right)
$$

where $\alpha_{k}(\mu, \omega)$ is given by (3.2).

In Kincaid [5], an exact expression for the $A_{\beta}^{1 / 2}$-norm of the SOR method was obtained for $\omega=\omega_{b}$ only. We state these results here for reference.

COROllary 3.2. If $A$ is a positive definite matrix of the form (1.1), then, for $m \geqq 1$,

$$
\left\|\mathfrak{L}_{\omega_{b}}^{m}\right\|_{A \beta^{1 / 2}}=\left(\omega_{b}-1\right)^{m}\left\{m \sigma_{\beta}\left(\bar{\mu}, \omega_{b}\right)+\left(m^{2} \sigma_{\beta}^{2}\left(\bar{\mu}, \omega_{b}\right)+1\right)^{1 / 2}\right\},
$$

where

$$
\sigma_{\beta}\left(\bar{\mu}, \omega_{b}\right) \equiv\left\{\left(2-\omega_{b}\right)^{2} /\left(\omega_{b}-1\right)+4(1-\beta)^{2} /\left(1-\bar{\mu}^{2} \beta^{2}\right)\right\}^{1 / 2} .
$$

By setting $\beta=0$ and $\beta=1$ in (3.4), the following well-established results for the $D^{1 / 2}$-norm and the $A^{1 / 2}$-norm, respectively, are obtained. Similar expressions can be found in Sheldon [6, p. 501], Golub and Varga [2, p. 161], Varga [7, p. 152], Young and Kincaid [9, pp. 31, 43], Kincaid [5, p. 20], and Young [11, pp. 248, 258].

COROllary 3.3. If $A$ is a positive definite matrix of the form (1.1), then, for $m \geqq 1$,

$$
\begin{aligned}
\left\|\mathscr{L}_{\omega_{b}}^{m}\right\|_{D^{1 / 2}} & =\left(\omega_{b}-1\right)^{m}\left\{m \sigma_{0}\left(\bar{\mu}, \omega_{b}\right)+\left(m^{2} \sigma_{0}^{2}\left(\bar{\mu}, \omega_{b}\right)+1\right)^{1 / 2}\right\}, \\
\left\|\mathscr{L}_{\omega_{b}}^{m}\right\|_{1^{1 / 2}} & =\left(\omega_{b}-1\right)^{m}\left\{m \sigma_{1}\left(\bar{\mu}, \omega_{b}\right)+\left(m^{2} \sigma_{1}^{2}\left(\bar{\mu}, \omega_{b}\right)+1\right)^{1 / 2}\right\}, \\
S\left(\mathscr{L}_{\omega_{b}}^{m}\right) & =\left(\omega_{b}-1\right)^{m},
\end{aligned}
$$

where

$$
\begin{aligned}
& \sigma_{n}\left(\bar{\mu}, \omega_{b}\right)=\frac{\omega_{h}}{\left(\omega_{h}-1\right)^{1 / \overline{2}}}=\frac{2}{\bar{\mu}}=\frac{1}{r^{1 / \overline{2}}}+r^{1 / 2}, \\
& \sigma_{1}\left(\bar{\mu}, \omega_{b}\right)=\frac{2-\omega_{b}}{\left(\omega_{h}-1\right)^{1 / 2}}=\frac{2}{\bar{\mu}}\left(1-\bar{\mu}^{2}\right)^{1 / 2}=\frac{1}{r^{1 / 2}}-r^{1 / 2},
\end{aligned}
$$

and $r=\omega_{b}-1$.

It is natural to inquire whether or not an exact expression for the $A_{\beta}^{1 / 2}$-norm can be found for values of $\omega$ other than the optimum value. We shall show that for a restricted range of values of $\omega$ the maximum in (3.1) always occurs at $\mu=\bar{\mu}$ for all $\beta, 0 \leqq \beta \leqq 1$, and $m \geqq 1$.

Without restricting $\omega, \mu$, or $\beta$, we are able to determine an exact expression for the $A_{\beta}^{1 / 2}$-norm of $\mathscr{L}_{\omega}^{m}$ only when $m=1$. Thus, we now determine $\left\|\mathfrak{L}_{\omega}\right\|_{A_{\beta^{1 / s}}}$ for $0<\omega<2$.

COROllary 3.4. If $A$ is a positive definite matrix of the form (1.1), then, for $0 \leqq \beta \leqq 1$,

$$
\left\|\mathfrak{L}_{\omega}\right\|_{A \beta^{1 / 2}}=\gamma_{\beta}(1, \bar{\mu}, \omega)+\left(\gamma_{\beta}^{2}(1, \bar{\mu}, \omega)+(1-\omega)^{2}\right)^{1 / 2}, \quad 0<\omega<2,
$$

where

$$
\gamma_{\beta}(1, \bar{\mu}, \omega)=\frac{1}{2}\left\{(2-\omega)^{2}+\frac{\omega^{2} \bar{\mu}^{2}(1-\beta)^{2}}{1-\bar{\mu}^{2} \beta^{2}}\right\}^{1 / 2} \omega \bar{\mu}
$$


Proof. We see that the right side of (3.1) is maximized whenever $\gamma_{\beta}(1, \mu, \omega)$ is maximized. Now, $\gamma_{\beta}(1, \mu, \omega)$ is an increasing function of $\mu$ for fixed $\omega$ and $\beta$. Therefore, $\gamma_{\beta}(1, \mu, \omega)$ assumes its maximum value at $\mu=\bar{\mu}$ for all $\mu$ such that $-\bar{\mu} \leqq \mu \leqq \bar{\mu}$. Since $\bar{\mu}$ is necessarily an eigenvalue of $B$, the right side of (3.1) is maximized at $\mu=\bar{\mu}$ for $\mu$ ranging over only the eigenvalues of $B$. Thus, from the theory established in Young and Kincaid [9] (see, also, Kincaid [5] or Young [11]), we have (3.8) for all $\omega$ such that $0<\omega<2$.

TABLE 3.1

Comparison of $\left\|\mathfrak{L}_{\omega}\right\|_{A \beta^{1 / 2}}$ for $0 \leqq \beta \leqq 1$ and $S\left(\mathfrak{L}_{\omega}\right)$ for $0<\omega<2$ where $\bar{\mu}=.9877$

\begin{tabular}{rrrrrrr}
\hline$\omega$ & $\beta=0$ & $\beta=.2$ & $\beta=.5$ & $\beta=.8$ & $\beta=1$ & $S\left(\mathfrak{L}_{\omega}\right)$ \\
\hline 0.0 & 1.0000 & 1.0000 & 1.0000 & 1.0000 & 1.0000 & 1.0000 \\
.1 & .9988 & .9988 & .9987 & .9987 & .9987 & .9887 \\
.2 & .9986 & .9982 & .9977 & .9974 & .9973 & .9973 \\
.3 & 1.0009 & .9992 & .9975 & .9963 & .9958 & .9957 \\
.4 & 1.0082 & 1.0035 & .9989 & .9957 & .9942 & .9939 \\
.5 & 1.0240 & 1.0137 & 1.0031 & .9960 & .9926 & .9918 \\
.6 & 1.0529 & 1.0327 & 1.0120 & .9979 & .9911 & .9895 \\
.7 & 1.0098 & 1.0644 & 1.0275 & 1.0020 & .9897 & .9868 \\
.8 & 1.1695 & 1.1124 & 1.0519 & 1.0094 & .9886 & .9837 \\
.9 & 1.2651 & 1.1798 & 1.0874 & 1.0210 & .9879 & .9800 \\
1.0 & 1.3882 & 1.2686 & 1.1359 & 1.0377 & .9877 & .9755 \\
1.1 & 1.5393 & 1.3798 & 1.1986 & 1.0604 & .9879 & .9701 \\
1.2 & 1.7181 & 1.5141 & 1.2769 & 1.0899 & .9886 & .9632 \\
1.3 & 1.9245 & 1.6719 & 1.3719 & 1.1273 & .9897 & .9543 \\
1.4 & 2.1584 & 1.8535 & 1.4851 & 1.1743 & .9911 & .9422 \\
1.5 & 2.4199 & 2.0597 & 1.6180 & 1.2331 & .9926 & .9245 \\
1.6 & 2.7090 & 2.2910 & 1.7721 & 1.3068 & .9942 & .8952 \\
1.7 & 3.0259 & 2.5476 & 1.9490 & 1.3991 & .9958 & .8262 \\
1.729 & 3.1245 & 2.6281 & 2.0056 & 1.4303 & .9962 & .7294 \\
1.8 & 3.3705 & 2.8299 & 2.1496 & 1.5137 & .9973 & .8000 \\
1.9 & 3.7430 & 3.1382 & 2.3747 & 1.6541 & .9987 & .9000 \\
2.0 & 4.1435 & 3.4724 & 2.6247 & 1.8221 & 1.0000 & 1.0000 \\
\hline & & & & & & \\
\hline
\end{tabular}

As a consequence of this corollary, we now obtain expressions for the $D^{1 / 2}$-norm and the $A^{1 / 2}$-norm of $\mathfrak{L}_{\omega}$ for $0<\omega<2$.

COROllary 3.5. If $A$ is a positive definite matrix of the form (1.1), then

$$
\begin{aligned}
\left\|\mathfrak{L}_{\omega}\right\|_{D^{1 / 2}} & =\gamma_{0}(1, \bar{\mu}, \omega)+\left(\gamma_{0}^{2}(1, \bar{\mu}, \omega)+(1-\omega)^{2}\right)^{1 / 2}, & & 0<\omega<2, \\
\left\|\mathscr{L}_{\omega}\right\|_{A^{1 / 2}} & =\gamma_{1}(1, \bar{\mu}, \omega)+\left(\gamma_{1}^{2}(1, \bar{\mu}, \omega)+(1-\omega)^{2}\right)^{1 / 2}, & & 0<\omega<2, \\
S\left(\mathfrak{L}_{\omega}\right) & =\left[\left(\frac{1}{2} \omega \mu\right)+\left(\left(\frac{1}{2} \omega \mu\right)^{2}-(\omega-1)\right)^{1 / 2}\right]^{2}, & & 0<\omega \leqq \omega_{b}, \\
& =\omega-1, & & \omega_{b} \leqq \omega \leqq 2,
\end{aligned}
$$

where 


$$
\gamma_{0}(1, \bar{\mu}, \omega)=\frac{1}{2} \omega \bar{\mu}\left((2-\omega)^{2}+\omega^{2} \bar{\mu}^{2}\right)^{1 / 2}, \quad \gamma_{1}(1, \bar{\mu}, \omega)=\frac{1}{2} \omega \bar{\mu}(2-\omega) .
$$

By comparing $S\left(\mathscr{L}_{\omega(2-\omega)}\right)$ given by (3.11), since $0 \leqq \omega(2-\omega) \leqq 1 \leqq \omega_{b}$, and $\left\|\mathscr{L}_{\omega}\right\|_{A^{1 / 2}}$ given by (3.10), we obtain the relation

$$
\left\|\mathfrak{L}_{\omega}\right\|_{A^{1 / 2}}^{2}=S\left(\mathfrak{L}_{\omega(2-\omega)}\right) \text {. }
$$

Young [10] establishes this result using a different approach. We note also that the $A^{1 / 2}$-norm is symmetric about $\omega=1$, so that

$$
\min _{0<\omega<2}\left\|\mathfrak{L}_{\omega}\right\|_{A^{1 / 2}}=\|\mathfrak{L}\|_{A^{1 / 2}}
$$

where $\mathscr{L} \equiv \mathfrak{L}$; however,

$$
\min _{0<\omega<2} S\left(\mathfrak{L}_{\omega}\right)=S\left(\mathfrak{L}_{\omega_{b}}\right) .
$$

Notice that while the $D^{1 / 2}$-norm of $\mathfrak{L}_{\omega}$ is not always less than unity, the $A^{1 / 2}$-norm and spectral radius are. This fact is illustrated in Table 3.1.

We now investigate norms of the SOR method for $m \geqq 2$. When $m \geqq 2$, the maximum in expression (3.1) does not necessarily occur at $\mu=\bar{\mu}$ for all $\omega$ such that $0<\omega<2$ and for all $\bar{\mu}$ such that $0<\bar{\mu}<1$, as was the case for $m=1$. Nevertheless, we shall show that for all $\omega$, such that $0<\omega \leqq \omega_{b}$, this maximum is obtained at $\mu=\bar{\mu}$ for all $\bar{\mu}$ such that $0<\bar{\mu}<1$. In fact, numerical results indicate that this maximum seems to occur at $\mu=\bar{\mu}$ for all $\bar{\mu}$ such that $0<\bar{\mu}<1$, whenever $\omega$ is restricted to the range $0<\omega<\omega_{b}+\epsilon(m, \beta, \bar{\mu})$ where $0<\epsilon(m, \beta, \bar{\mu})<2-\omega_{b}$. Here, the exact value of $\epsilon$ depends on $m, \beta$, and $\bar{\mu}$. Moreover, numerical results indicate that this maximum seems to occur at $\mu=\bar{\mu}$ for many particular values of $\bar{\mu}$ near unity and many selected values of $m$ without restrictions on $\omega$ or $\beta$.

In order to establish an exact expression for the $A_{\beta}^{1 / 2}$-norm of $\mathscr{L}_{\omega}^{m}$ for $0<\omega \leqq \omega_{b}$, we first show that

$$
\max _{\mu}\left|\alpha_{k}(\mu, \omega)\right|=\alpha_{k}(\bar{\mu}, \omega), \quad 0<\omega \leqq \omega_{b},
$$

where $\alpha_{k}(\mu, \omega)$ is defined by (3.2) for all $k \geqq 0$. Moreover,

$$
\alpha_{k}\left(\bar{\mu}, \omega_{b}\right)=(k+1)\left(\omega_{b}-1\right)^{k / 2} .
$$

For each fixed $\mu$ and $\omega, \alpha_{k}(\mu, \omega)$ as defined by (3.2) is a linear difference equation with constant coefficients which can be solved by assuming solutions of the form $\lambda^{k}(\mu, \omega)$. It can be easily verified that, for $k \geqq 0$,

$$
\begin{aligned}
\alpha_{k}(\mu, \omega)=\sum_{i=1}^{k} \lambda_{1}^{k-i} \lambda_{2}^{i} & =\frac{\lambda_{1}^{k+1}-\lambda_{2}^{k+1}}{\lambda_{1}-\lambda_{2}}, & & \lambda_{1} \neq \lambda_{2}, \\
& =(k+1) \lambda_{1}^{k}, & & \lambda_{1}=\lambda_{2},
\end{aligned}
$$

where $\lambda_{1} \equiv \lambda_{1}(\mu, \omega), \lambda_{2} \equiv \lambda_{2}(\mu, \omega)$ are the roots of $\lambda^{2}-(\omega \mu) \lambda+(\omega-1)=0$. We designate

$$
\begin{aligned}
& \lambda_{1}=\left(\frac{1}{2} \omega \mu\right)+\left(\left(\frac{1}{2} \omega \mu\right)^{2}-(\omega-1)\right)^{1 / 2} \\
& \lambda_{2}=\left(\frac{1}{2} \omega \mu\right)-\left(\left(\frac{1}{2} \omega \mu\right)^{2}-(\omega-1)\right)^{1 / 2}
\end{aligned}
$$

Hence, 


$$
\lambda_{1}+\lambda_{2}=\omega \mu, \quad \lambda_{1} \lambda_{2}=\omega-1 .
$$

Suppose that $\omega$ is fixed such that $0<\omega<2$. Notice that $\lambda_{1}(-\mu, \omega)=-\lambda_{2}(\mu, \omega)$ and $\lambda_{2}(-\mu, \omega)=-\lambda_{1}(\mu, \omega)$ by (3.15) so that $\alpha_{k}(-\mu, \omega)=(-1)^{k} \alpha_{k}(\mu, \omega)$ by (3.14). Clearly, $\left|\alpha_{k}(-\mu, \omega)\right|=\left|\alpha_{k}(\mu, \omega)\right|$. Therefore, we need only consider nonnegative values of $\mu$.

Suppose that $0<\omega<1$. Whenever $\mu \neq 0$, we note by (3.2) that $\alpha_{k}(\mu, \omega)$ is a polynomial in $\mu$ with positive coefficients. Therefore, $\alpha_{k}$ is an increasing function of $\mu$ and it assumes its maximum value over the interval $0<\mu \leqq \bar{\mu}$ at $\mu=\bar{\mu}$. If $\mu=0$, then $0 \leqq\left|\alpha_{k}(0, \omega)\right|<\left|\alpha_{k}(\bar{\mu}, \omega)\right|$. Thus, (3.12) holds for $0<\omega<1$.

Suppose that $\omega=1$. Then, by (3.2), we have $\alpha_{k}(\mu, 1)=\mu^{k}$ for all $k$ and (3.12) follows immediately.

Suppose that $\omega=\omega_{b}$. We define $\theta$ by $\mu=\bar{\mu} \cos \theta, 0 \leqq \theta \leqq \pi / 2$. It is easy to show that $\lambda_{1}=\left(\omega_{b}-1\right)^{1 / 2} e^{i \theta}$ and $\lambda_{2}=\left(\omega_{b}-1\right)^{1 / 2} e^{-i \theta}$ from (3.15), using (3.16). Therefore, by (3.14), we have, for $k \geqq 0$,

$$
\begin{aligned}
\alpha_{k}\left(\mu, \omega_{b}\right) & =\left(\omega_{b}-1\right)^{k / 2}(k+1), & & \mu=\bar{\mu}(\theta=0), \\
& =\left(\omega_{b}-1\right)^{k / 2} \frac{\sin (k+1) \theta}{\sin \theta}, & & 0 \leqq \mu<\bar{\mu}(0<\theta \leqq \pi / 2) .
\end{aligned}
$$

By mathematical induction for any value of $\theta$, we have

$$
\left|\frac{\sin (k+1) \theta}{\sin \theta}\right| \leqq(k+1)
$$

(see, for instance, Golub [1]). Therefore, for any $\mu$ such that $0 \leqq \mu \leqq \bar{\mu}$, we have, by (3.17), $\left|\alpha_{k}\left(\mu, \omega_{b}\right)\right| \leqq\left(\omega_{b}-1\right)^{k / 2}(k+1)$. Moreover, (3.13) holds and, therefore, (3.12) follows for $\omega=\omega_{b}$.

Suppose that $1<\omega \leqq \omega_{b}$. We now show that

$$
\max _{0<\mu \leq \bar{\mu}}\left|\alpha_{k}(\mu, \omega)\right|=\alpha_{k}(\bar{\mu}, \omega) \text {. }
$$

Since $2(\omega-1)^{1 / 2} / \omega$ is an increasing function of $\omega$ in the range $1<\omega \leqq \omega_{b}$ and $2\left(\omega_{b}-1\right)^{1 / 2} / \omega_{b}=\bar{\mu}$, we have $0<2(\omega-1)^{1 / 2} / \omega \leqq \bar{\mu}$. Define $\theta$ by

$$
\mu=\left(2(\omega-1)^{1 / 2} \omega\right) \cos \theta, \quad 0 \leqq \theta<\pi / 2,
$$

for $0<\mu \leqq 2(\omega-1)^{1 / 2} / \omega$ and define $\psi$ by

$$
\mu=\left(2(\omega-1)^{1 / 2} / \omega\right) \cosh \psi, \quad 0 \leqq \psi \leqq \cosh ^{-1}\left(\omega \bar{\mu} / 2(\omega-1)^{1 / 2}\right)
$$

for $\mu \geqq 2(\omega-1)^{1 / 2} / \omega$. One can easily verify that for any value of $\mu$ such that $0<\mu \leqq \bar{\mu}$ and $1<\omega \leqq \omega_{b}$ (see, for instance, Householder [3])

$$
\begin{aligned}
\alpha_{k}(\mu, \omega) & =(\omega-1)^{k / 2} \frac{\sin (k+1) \theta}{\sin \theta}, & & 0<\mu<\frac{2(\omega-1)^{1 / 2}}{\omega}<\bar{\mu}, \\
& =(\omega-1)^{k / 2}(k+1), & & 0<\mu=\frac{2(\omega-1)^{1 / 2}}{\omega}<\bar{\mu}, \\
& =(\omega-1)^{k / 2} \frac{\sinh (k+1) \psi}{\sinh \psi}, & & 0<\frac{2(\omega-1)^{1 / 2}}{\omega}<\mu \leqq \bar{\mu} .
\end{aligned}
$$


By (3.18) and (3.20), we have

$$
0<\alpha_{k}(\mu, \omega) \leqq(\omega-1)^{k / 2}(k+1), \quad 0<\mu \leqq 2(\omega-1)^{1 / 2} / \omega .
$$

Moreover, by induction it can be shown that, for any value of $\psi \geqq 0$,

$$
\frac{\sinh (k+1) \psi}{\sinh \psi} \geqq(k+1) .
$$

Therefore, we have by (3.20)

$$
\alpha_{k}(\mu, \omega) \geqq(\omega-1)^{k / 2}(k+1), \quad 2(\omega-1)^{1 / 2} / \omega \leqq \mu \leqq \bar{\mu} .
$$

By (3.22) and (3.23), we observe that the maximum of $\alpha_{k}(\mu, \omega)$ occurs when $\mu$ has a value such that $2(\omega-1)^{1 / 2} / \omega \leqq \mu \leqq \bar{\mu}$. Moreover, $\sinh (k+1) \psi / \sinh \psi$ is an increasing function of $\psi$ so that by (3.20) the maximum value of $\alpha_{k}(\mu, \omega)$ occurs when $\psi$ is largest. But when $\psi$ is largest, $\mu$ is also. Hence, (3.19) follows. When $\mu=0$, we have by (3.2) that

$$
\begin{aligned}
\alpha_{k}(0, \omega) & =0, & & k \text { odd, } \\
& =(1-\omega)^{k / 2}, & & k \text { even, }
\end{aligned}
$$

and $0 \leqq\left|\alpha_{k}(0, \omega)\right|<(\omega-1)^{k / 2}(k+1)$. Hence, (3.12) follows for $1<\omega \leqq \omega_{b}$.

By (3.12) and Theorem 3.1, we have

$$
\max _{-\bar{\mu} \leqq \mu \leqq \bar{\mu}} \gamma_{\beta}(m, \mu, \omega)=\gamma_{\beta}(m, \bar{\mu}, \omega), \quad 0<\omega \leqq \omega_{b} .
$$

Hence, we have established the following corollary.

COROLlaRY 3.6. If $A$ is a positive definite matrix of the form (1.1), then, for $m \geqq 1$ and $0 \leqq \beta \leqq 1$,

$$
\left\|\mathscr{L}_{\omega}^{m}\right\|_{A_{\beta^{1} / 2}}=\gamma_{\beta}(m, \bar{\mu}, \omega)+\left(\gamma_{\beta}^{2}(m, \bar{\mu}, \omega)+(1-\omega)^{2 m}\right)^{1 / 2}, \quad 0<\omega \leqq \omega_{b},
$$

where

$$
\gamma_{\beta}(m, \bar{\mu}, \omega)=\frac{1}{2}\left\{(2-\omega)^{2}+\frac{\omega^{2} \bar{\mu}^{2}(1-\beta)^{2}}{1-\bar{\mu}^{2} \beta^{2}}\right\}^{1 / 2} \alpha_{2 m-1}(\bar{\mu}, \omega),
$$

and $\alpha_{k}$ is defined by (3.2).

As an immediate consequence, we have the following corollary:

COROllary 3.7. If $A$ is a positive definite matrix of the form (1.1), then, for $m \geqq 1$ and $0 \leqq \beta \leqq 1$,

$$
\begin{array}{ll}
\left\|\mathfrak{L}_{\omega}^{m}\right\|_{D^{1 / 2}}=\gamma_{0}(m, \bar{\mu}, \omega)+\left(\gamma_{0}^{2}(m, \bar{\mu}, \omega)+(1-\omega)^{2 m}\right)^{1 / 2}, & 0<\omega \leqq \omega_{b}, \\
\left\|\mathfrak{L}_{\omega}^{m}\right\|_{A^{1 / 2}}=\gamma_{1}(m, \bar{\mu}, \omega)+\left(\gamma_{1}^{2}(m, \bar{\mu}, \omega)+(1-\omega)^{2 m}\right)^{1 / 2}, & 0<\omega \leqq \omega_{b},
\end{array}
$$

where

$$
\begin{aligned}
& \gamma_{0}(m, \bar{\mu}, \omega)=\frac{1}{2}\left((2-\omega)^{2}+\omega^{2} \bar{\mu}^{2}\right)^{1 / 2}\left|\alpha_{2 m-1}(\bar{\mu}, \omega)\right|, \\
& \gamma_{1}(m, \bar{\mu}, \omega)=\frac{1}{2}(2-\omega)\left|\alpha_{2 m-1}(\bar{\mu}, \omega)\right| .
\end{aligned}
$$

Now, we see that, for $m \geqq 1$,

$$
\min _{0 \leqq \beta \leqq 1}\left\|\mathfrak{L}_{\omega}^{m}\right\|_{A^{1 / 2}}=\left\|\mathfrak{L}_{\omega}^{m}\right\|_{A^{1 / 2}}, \quad 0<\omega \leqq \omega_{b} .
$$


Hence, the $A^{1 / 2}$-norm is the "most desirable" of the $A_{\beta}^{1 / 2}$-norm for the SOR method with $0<\omega \leqq \omega_{b}$.

Numerical results indicate that

$$
\min _{0<\omega<2}\left\|\mathfrak{L}_{\omega}^{m}\right\|_{A^{1 / 2}}=\left\|\mathfrak{L}_{\omega_{b} \pm \epsilon(m, \tilde{\mu})}^{m}\right\|_{A^{1 / 2}},
$$

where $1 \leqq \omega_{b} \pm \epsilon(m, \bar{\mu})<2$ (see Table 3.2). For $\bar{\mu}$ fixed, the omega value that minimizes the $A^{1 / 2}$-norm is a function of $m$. By numerical experiments, we note that, for the first few iterations, this "optimum" omega is less than $\omega_{b}$ and, from then on, always greater than $\omega_{b}$. As $m$ increases, it seems to approach $\omega_{b}$ from above.

For the $D^{1 / 2}$-norm, numerical studies show that

$$
\min _{0<\omega<2}\left\|\mathscr{L}_{\omega}^{m}\right\|_{D^{1 / 2}}=\left\|\mathcal{L}_{\omega_{b t \in(m, \bar{\mu})}^{m}}\right\|_{D^{1 / 2}},
$$

where $0<\omega_{b} \pm \epsilon(m, \bar{\mu})<2$ (see Table 3.2). For fixed $\bar{\mu}$, the "optimum" omega with respect to the $D^{1 / 2}$-norm varies with $m$. These values of omega are initially less than one but finally, after several iterations, greater than $\omega_{b}$.

TABLE 3.2

\begin{tabular}{|c|c|c|}
\hline$m$ & $\beta=0$ & $\beta=1$ \\
\hline 1 & .200 & 1.000 \\
\hline 2 & .225 & 1.400 \\
\hline 3 & .237 & 1.562 \\
\hline 4 & .275 & 1.643 \\
\hline 5 & .325 & 1.694 \\
\hline 6 & .400 & 1.728 \\
\hline 7 & .475 & 1.747 \\
\hline 8 & .525 & 1.759 \\
\hline 9 & .587 & 1.762 \\
\hline 10 & .662 & 1.759 \\
\hline 11 & .737 & 1.739 \\
\hline 12 & .825 & 1.723 \\
\hline 13 & 1.719 & 1.717 \\
\hline 14 & 1.713 & 1.712 \\
\hline 15 & $1.704 *$ & 1.703 \\
\hline 16 & $1.708 *$ & $1.699 *$ \\
\hline 17 & $1.697^{*}$ & $1.697^{*}$ \\
\hline 18 & 1.796 & 1.694 \\
\hline 19 & 1.690 & 1.691 \\
\hline 20 & 1.680 & 1.689 \\
\hline 21 & 1.687 & 1.687 \\
\hline 22 & 1.684 & 1.684 \\
\hline 23 & $1.739 *$ & $1.684^{*}$ \\
\hline 24 & $1.683^{*}$ & $1.684 *$ \\
\hline 25 & 1.681 & 1.681 \\
\hline
\end{tabular}

Values of omega at which $\min _{0<\omega<2}\left\|\mathfrak{L}_{\omega}^{m}\right\|_{A_{\beta}{ }^{1 / 2}}$ occurs for $\bar{\mu}=.98$ and $\omega_{\iota}=1.668$

\footnotetext{
* Indicates that in formula (3.1) the maximum did not occur at $\mu=\bar{\mu}$.
} 
We now show that (3.25) does not hold for all $\omega$ such that $0<\omega<2$. We are able to establish that for $\beta=0$ the right side of (3.1) is not maximized at $\mu=\bar{\mu}$ for all $\omega$ such that $0<\omega<2$ and all $\bar{\mu}$ such that $0<\bar{\mu}<1$, i.e., that the spectral norm of $M_{m}(\mu, \omega)$ is not largest when $\mu=\bar{\mu}$. Numerical results indicate that, for various values of $m, \omega$ must be restricted to the range $0<\omega \leqq \omega_{b}$, in order for th? $D^{1 / 2}$-norm of $\mathfrak{L}_{\omega}^{m}$ to be given by (3.25) for all $\bar{\mu}$ such that $0<\bar{\mu}<1$. We now show by an example that if $\bar{\mu}$ and/or $m$ are not restricted, then (3.25) does not follow for all $\omega$ such that $\omega_{b}<\omega<2$. Consider the case $m=2, \bar{\mu}$ fixed such that $\bar{\mu}^{2}<\frac{1}{2}$, and $\omega=\alpha$ such that $\alpha>1$ and $\bar{\mu}^{2}=2(\alpha-1) / \alpha^{2}$. Note that $\alpha_{3}(\bar{\mu}, \alpha)=0$ since $\alpha_{3}(\mu, \omega)=$ $\omega \mu\left(\omega^{2} \mu^{2}-2(\omega-1)\right)$. Since $4\left(\omega_{b}-1\right) / \omega_{b}^{2}=\ddot{\mu}^{2}=2(\alpha-1) / \alpha^{2}<4(\alpha-1) / \alpha^{2}$ and $4(\omega-1) / \omega^{2}$ is an increasing function of $\omega$, we see that $\alpha>\omega_{b}$. Clearly, for any other nonzero eigenvalue $\mu$ such that $|\mu| \neq \bar{\mu},\left|\alpha_{3}(\mu, \alpha)\right|>0=\left|\alpha_{3}(\bar{\mu}, \alpha)\right|$. Moreover, $\gamma_{\beta}(2, \mu, \alpha)>0=\gamma_{\beta}(2, \bar{\mu}, \alpha)$ for any nonzero eigenvalue $\mu$ such that $|\mu| \neq \bar{\mu}$. Hence, the right side of (3.1) does not assume its maximum value at $\mu=\bar{\mu}$ for the case $\beta=0, m=2$, and $\omega=\alpha$. The following numerical example illustrates this situation (see also Table 3.3).

TABLE 3.3

Values of $\mu$ in the range $-\bar{\mu} \leqq \mu \leqq \bar{\mu}$ at which (3.1) is maximized for the case $m=2, \bar{\mu}=.7$, and $1 \leqq \omega<2$

\begin{tabular}{lll}
\multicolumn{1}{c}{$\omega$} & $\beta=0$ & $\beta=1$ \\
\hline 1.0 & .700 & .700 \\
1.1 & .700 & .700 \\
1.167 & .700 & .700 \\
1.2 & .700 & .700 \\
1.3 & .700 & .700 \\
1.4 & .420 & .373 \\
1.5 & .443 & .373 \\
1.6 & .467 & .397 \\
1.7 & .490 & .397 \\
1.8 & .490 & .397 \\
1.9 & .490 & .397 \\
2.0 & .490 & .327
\end{tabular}

Consider the rectangle with sides $a=3 h$ and $b=4 h$, where $h$ is the mesh size, e.g., $1.0 \times 1.5$ with $h=\frac{1}{3}$. According to Young [11], the spectral radius of the Jacobi matrix $B$ is given by

$$
\bar{\mu}=\frac{1}{2}\left(\cos \frac{\pi h}{a}+\cos \frac{\pi h}{b}\right)=\frac{1}{4}\left(1+2^{1 / 2}\right) \doteq .603 .
$$

Hence, $\bar{\mu}^{2} \doteq .364$ and $\omega_{b}=2 /\left(1+\left(1-\bar{\mu}^{2}\right)^{1 / 2}\right) \doteq 1.11$. Choosing $\alpha=$ $1+\left(1-2 \bar{\mu}^{2}\right)^{1 / 2}=1.522$, we are ready to determine which eigenvalue of $B$ maximizes the right side of (3.1) with $\beta=0$. The eigenvalues of $B$ are given by

$$
\mu_{p, i}=\frac{1}{2}\left(\cos \frac{p \pi h}{a}+\cos \frac{q \pi h}{b}\right), \quad p=1,2, q=1,2,3,
$$


which are $\pm \frac{1}{4}\left(1+2^{1 / 2}\right) \doteq \pm .603, \pm \frac{1}{4}= \pm .25, \pm \frac{1}{4}\left(1-2^{1 / 2}\right) \doteq \pm .103$. Since $\alpha_{3}(\mu, \omega)=\omega \mu\left(\omega^{2} \mu^{2}-2(\omega-1)\right)$ and $\gamma_{0}(2, \mu, \omega)=\frac{1}{2}\left\{(2-\omega)^{2}+\omega^{2} \mu^{2}\right\}^{1 / 2}\left|\alpha_{3}(\mu, \omega)\right|$, we have $\left|\alpha_{3}( \pm .603,1.522)\right| \doteq 0,\left|\alpha_{3}( \pm .25,1.522)\right| \doteq .342,\left|\alpha_{3}( \pm .103,1.522)\right|=.159$ and $\gamma_{0}(2, \pm .603,1.522) \doteq 0, \gamma_{0}(2, \pm .25,1.522) \doteq .104, \gamma_{0}(2, \pm .103,1.522) \doteq .04$. Hence,

$$
\left\|\mathscr{L}_{\alpha}^{2}\right\|_{D^{1 / 2}}=\gamma_{0}\left(2, \mu_{\alpha}, \alpha\right)+\left(\gamma_{0}^{2}\left(2, \mu_{\alpha}, \alpha\right)+(1-\alpha)^{2}\right)^{1 / 2},
$$

where $\alpha \doteq 1.522$ and $\mu_{\alpha}= \pm .25$ which is not $\bar{\mu}$ and does not agree with (3.25) since $\alpha>\omega_{b}$. These results seem to reveal a slight oversight by Wachspress [8, p. 130]. We shall collate our results with his results in the next section.

4. Approximate Norm Expressions. We now develop approximate expressions for the $D^{1 / 2}$-norm and the $A^{1 / 2}$-norm of $\mathcal{L}_{\omega}^{m}$. This analysis is based on work by Wachspress [8]; however, the more compact notation of Young and Kincaid [9] is used (see, also, Kincaid [5] or Young [11]).

THEOREM 4.1. If $A$ is a positive definite matrix of the form (1.1), then, for $m \geqq 1$ and $0<\omega \leqq \omega_{b}$,

$$
\begin{aligned}
&\left.|| \mathcal{L}_{\omega}^{m}\right|_{D^{1 / 2}} \approx\left|\alpha_{2 m-1}(\bar{\mu}, \omega)\right|\left\{\left[1+\left|\gamma_{a}(\bar{\mu}, \omega)\right|\right]\left[1+(\omega-1)^{2}\left|\gamma_{a}(\bar{\mu}, \omega)\right|^{-1}\right]\right\}^{1 / 2}, \\
&|| \mathcal{L}_{\omega}^{m}||_{A^{1 / 2}} \approx\left|\alpha_{2 m-1}(\bar{\mu}, \omega)\right|(2-\omega),
\end{aligned}
$$

where

$$
\gamma_{a}(\bar{\mu}, \omega)=\left[\left(\frac{1}{2} \omega \bar{\mu}\right)+\left(\left(\frac{1}{2} \omega \bar{\mu}\right)^{2}-(\omega-1)\right)^{1 / 2}\right]^{2} .
$$

Proof. From (3.3) with $\beta=0$ and Corollary 3.7, the $D^{1 / 2}$-norm of $\mathscr{L}_{\omega}^{m}$ is just the spectral norm of

$$
M_{m}(\bar{\mu}, \omega)=\left(\begin{array}{cc}
(1-\omega) \alpha_{2 m-2}(\bar{\mu}, \omega) & \alpha_{2 m-1}(\bar{\mu}, \omega) \\
(1-\omega) \alpha_{2 m-1}(\bar{\mu}, \omega) & \alpha_{2 m}(\bar{\mu}, \omega)
\end{array}\right) .
$$

(For details, see Kincaid [5] or Young [11].)

Following Wachspress [8], we now obtain approximate expressions that relate $\alpha_{2 m-2}$ and $\alpha_{2 m}$ to $\alpha_{2 m-1}$. Let

$$
\begin{aligned}
& \gamma_{a}^{1 / 2} \equiv \gamma_{a}^{1 / 2}(\mu, \omega)=\left(\frac{1}{2} \omega \mu\right)+\left(\left(\frac{1}{2} \omega \mu\right)^{2}-(\omega-1)\right)^{1 / 2}, \\
& \gamma_{b}^{1 / 2} \equiv \gamma_{b}^{1 / 2}(\mu, \omega)=\left(\frac{1}{2} \omega \mu\right)-\left(\left(\frac{1}{2} \omega \mu\right)^{2}-(\omega-1)\right)^{1 / 2}
\end{aligned}
$$

We also define

$$
\epsilon \equiv \epsilon(\mu, \omega)=\left(1-\frac{4(\omega-1)}{\omega^{2} \mu^{2}}\right)^{1 / 2}, \quad r \equiv r(\mu, \omega)=\frac{1-\epsilon}{1+\epsilon} .
$$

Therefore, it follows that

$$
\begin{aligned}
\gamma_{a}^{1 / 2} & =\frac{1}{2} \omega \mu(1+\epsilon), & \gamma_{b}^{1 / 2} & =\frac{1}{2} \omega \mu(1-\epsilon), \\
\gamma_{a} & =\omega^{2} \mu^{2} /(1+r)^{2}, & \gamma_{b} & =r^{2} \omega^{2} \mu^{2} /(1+r)^{2}=r^{2} \gamma_{a} .
\end{aligned}
$$

From (3.14), we have

$$
\alpha_{k} \equiv \alpha_{k}(\mu, \omega)=\sum_{i=0}^{k} \gamma_{a}^{(k-i) / 2} \gamma_{b}^{i / 2}
$$


Thus, we see that

$$
\alpha_{k}=\gamma_{a}^{k / 2}\left(1+r+r^{2}+\cdots+r^{k-1}+r^{k}\right) .
$$

Hence, we obtain the following approximations:

$$
\alpha_{2 m-2} \approx \gamma_{a}^{-1 / 2} \alpha_{2 m-1}, \quad \alpha_{2 m} \approx \gamma_{a}^{1 / 2} \alpha_{2 m-1} .
$$

Therefore, we approximate $M_{m}(\bar{\mu}, \omega)$ by the matrix

$$
Q_{m}(\bar{\mu}, \omega) \equiv \alpha_{2 m-1}(\bar{\mu}, \omega)\left(\begin{array}{cc}
(1-\omega) \gamma_{a}^{-1 / 2}(\bar{\mu}, \omega) & 1 \\
(1-\omega) & \gamma_{a}^{1 / 2}(\bar{\mu}, \omega)
\end{array}\right) .
$$

Since $\gamma_{a}(\bar{\mu}, \omega)$ may be complex-valued, we are interested in determining $S\left(Q_{m}(\bar{\mu}, \omega) Q_{m}^{H}(\bar{\mu}, \omega)\right)$. The characteristic equation is, of course,

$$
\Gamma^{2}-T_{m}(\bar{\mu}, \omega) \Gamma+\Delta_{m}=0,
$$

where

$$
\begin{aligned}
T_{m}(\bar{\mu}, \omega) & =\operatorname{trace} Q_{m}(\bar{\mu}, \omega) Q_{m}^{H}(\bar{\mu}, \omega) \\
& =\left|\alpha_{2 m-1}(\bar{\mu}, \omega)\right|^{2}\left[1+\left|\gamma_{a}(\bar{\mu}, \omega)\right|\right]\left[1+(1-\omega)^{2}\left|\gamma_{a}(\bar{\mu}, \omega)\right|^{-1}\right] \\
\Delta_{m} & =\operatorname{det} Q_{m}(\bar{\mu}, \omega) Q_{m}^{H}(\bar{\mu}, \omega)=0 .
\end{aligned}
$$

Hence,

$$
\left\|\mathfrak{L}_{\omega}^{m}\right\|_{D^{1 / 2}} \approx\left\{T_{m}(\bar{\mu}, \omega)\right\}^{1 / 2}
$$

and (4.1) follows.

We now perform a similar analysis to obtain an approximation for the $A^{1 / 2}$-norm formula. Recall from Kincaid [5] or Young [11] that the relevant $2 \times 2$ scalar matrix is

$$
V_{m}(\bar{\mu}, \omega)=\left(\begin{array}{ll}
\alpha_{2 m}-\frac{\omega \bar{\mu}}{2} \alpha_{2 m-1} & \left(1-\frac{\omega p \bar{\mu}}{2}\right) \alpha_{2 m-1} \\
\left(1-\frac{\omega \bar{\mu}}{2 p}\right) \alpha_{2 m-1} & \alpha_{2 m}-\frac{\omega \bar{\mu}}{2} \alpha_{2 m-1}
\end{array}\right)
$$

where $\beta=1$ and

$$
p+1 / p=2 / \bar{\mu}, \quad p^{2}+1 / p^{2}=4 / \bar{\mu}^{2}-2 .
$$

Again, we apply the approximations (4.4) to approximate $V_{m}(\bar{\mu}, \omega)$ by the matrix

$$
\begin{aligned}
U_{m}(\bar{\mu}, \omega) & =\alpha_{2 m-1}\left(\begin{array}{cc}
\gamma_{a}^{1 / 2}-\omega \bar{\mu} / 2 & 1-\omega \bar{\mu} p / 2 \\
1-\omega \bar{\mu} / 2 p & \gamma_{a}^{1 / 2}-\omega \bar{\mu} / 2
\end{array}\right) \\
& =\alpha_{2 m-1}\left(\begin{array}{cc}
\omega \bar{\mu} \epsilon / 2 & 1-\omega \bar{\mu} p / 2 \\
1-\omega \bar{\mu} / 2 p & \omega \bar{\mu} \epsilon / 2
\end{array}\right)
\end{aligned}
$$

where $\alpha_{2 m-1} \equiv \alpha_{2 m-1}(\bar{\mu}, \omega)$, using (4.3). The relevant characteristic equation is again (4.5) where

$$
T_{m}(\bar{\mu}, \omega)=\operatorname{trace} U_{m}(\bar{\mu}, \omega) U_{m}^{H}(\bar{\mu}, \omega), \quad \Delta_{m}=\operatorname{det} U_{m}(\bar{\mu}, \omega) U_{m}^{H}(\bar{\mu}, \omega) .
$$

Hence, we have from (4.6) 


$$
\text { det } \begin{aligned}
U_{m}(\bar{\mu}, \omega) & =\alpha_{2 m-1}^{2}\left\{\frac{\omega^{2} \bar{\mu}^{2}}{4} \epsilon^{2}-\left(1-\frac{\omega \bar{\mu}}{2 p}\right)\left(1-\frac{\omega \bar{\mu} p}{2}\right)\right\} \\
& =\alpha_{2 m-1}^{2}\left\{\frac{\omega^{2} \bar{\mu}^{2}}{4}\left(\epsilon^{2}-1\right)-(1-\omega)\right\}=0
\end{aligned}
$$

using (4.3). Therefore, $\Delta_{m}=0$. Moreover, we note that

$$
\begin{aligned}
T_{m}(\bar{\mu}, \omega) & =\left|\alpha_{2 m-1}\right|^{2}\left\{\frac{\omega^{2} \bar{\mu}^{2}}{2} \epsilon^{2}+\left(1-\frac{\omega \bar{\mu}}{2 p}\right)^{2}+\left(1-\frac{\omega \bar{\mu} p}{2}\right)^{2}\right\} \\
& =\left|\alpha_{2 m-1}\right|^{2}\left\{(\omega-1)^{2}+\frac{\omega^{2} \bar{\mu}^{2}}{2}\left(\epsilon^{2}-1\right)+1\right\} \\
& =\left|\alpha_{2 m-1}\right|^{2}(\omega-2)^{2},
\end{aligned}
$$

since, by (4.6),

$$
(1-\omega \bar{\mu} / 2 p)^{2}+(1-\omega \bar{\mu} p / 2)^{2}=(\omega-1)^{2}-\omega^{2} \bar{\mu}^{2} / 2+1 .
$$

Hence, $\left\|\mathfrak{L}_{\omega}^{m}\right\|_{A^{1 / 2}} \approx\left\{T_{m}(\bar{\mu}, \omega)\right\}^{1 / 2}$ and (4.2) follows.

Equation (4.1) appears in Wachspress [8, p. 131] in a slightly different form. We note also that the restriction $0<\omega \leqq \omega_{b}$ must be added as previously mentioned.

As an immediate consequence, we obtain the following corollary.

COROLlaRY 4.2. If $A$ is a positive definite matrix of the form (1.1), then, for $m \geqq 1$,

$$
\begin{aligned}
\left\|\mathfrak{L}_{\omega_{b}}^{m}\right\|_{r^{1 / 2}} & \approx\left|\alpha_{2 m-1}\left(\bar{\mu}, \omega_{b}\right)\right| \omega_{b}=2 m \sigma_{0}\left(\bar{\mu}, \omega_{b}\right)\left(\omega_{b}-1\right)^{m} \\
& =4 m\left(\omega_{b}-1\right)^{m} / \bar{\mu}=2 m r^{m-1 / 2}(1+r), \\
\left\|\mathcal{L}_{\omega_{b}}^{m}\right\|_{A^{1 / 2}} \approx & :\left|\alpha_{2 m-1}\left(\bar{\mu}, \omega_{b}\right)\right|\left(2-\omega_{b}\right)=2 m \sigma_{1}\left(\bar{\mu}, \omega_{b}\right)\left(\omega_{b}-1\right)^{m} \\
= & 4 m\left(\omega_{b}-1\right)^{m}\left(1-\bar{\mu}^{2}\right)^{1 / 2} / \bar{\mu}=2 m r^{m-1 / 2}(1-r)
\end{aligned}
$$

where $r=\omega_{b}-1$.

Notice the agreement between Corollary 3.3 and Corollary 4.2. The expression (4.7) is given by Wachspress [8, p. 132].

By letting $\omega=1$ in Theorem 4.1, we obtain the following results which agree with Corollary 3.7. In fact, by Corollary 3.7 ; we observe that we obtain the exact norm formulas.

COROLlary 4.3. If $A$ is a positive definite matrix of the form (1.1), then

$$
\begin{aligned}
& || \mathfrak{L}^{m} \|_{D^{1 / 2}} \approx\left|\alpha_{2 m-1}(\bar{\mu}, 1)\right|\left(1+\bar{\mu}^{2}\right)^{1 / 2}=\bar{\mu}^{2 m-1}\left(1+\bar{\mu}^{2}\right)^{1 / 2}, \\
& \left\|\mathfrak{L}^{m}\right\|_{A^{1 / 2}} \approx\left|\alpha_{2 m-1}(\bar{\mu}, 1)\right|=\bar{\mu}^{2 m-1} .
\end{aligned}
$$

As noted by Wachspress [8], numerical studies indicate that if a significant error reduction is required, it is best to iterate a sufficient number of times with $\omega=\omega_{b}$ to accomplish this objective. It was shown in Kincaid [5] and in Young [11] that methods superior (in norm) to the ordinary SOR method can be obtained by either adopting a special procedure for obtaining the first iterant or by allowing the relaxation parameter to vary in a schematic manner. 
Acknowledgement. This paper includes work from the doctoral thesis of the author, who wishes to thank his supervisory professor Dr. David M. Young of The University of Texas at Austin.

Center for Numerical Analysis

The University of Texas at Austin

Austin, Texas 78712

1. Gene H. Golub, The Use of Chebyshev Matrix Polynomials in the Iterative Solution of Linear Systems Compared With the Methods of Successive Overrelaxation, Doctoral Thesis, University of Illinois, Urbana, Ill., 1959.

2. G. H. Golub \& R. S. VARGA, "Chebyshev semi-iterative methods, successive overrelaxation iterative methods, and second-order Richardson iterative methods. I, II," Numer. Math., v. 3, 1961, pp. 147-168. MR 26 \#3207; \#3208.

3. A. S. Householder, "The approximate solution of matrix problems," J. Assoc. Comput. Mach., v. 5, 1958, pp. 205-243. MR 23 \#B1646.

4. David R. Kincaid, An Analysis of a Class of Norms of Iterative Methods for Systems of Linear Equations, Doctoral Thesis, The University of Texas at Austin, 1971.

5. David R. Kincaid, A Class of Norms of Iterative Methods for Solving Systems of Linear Equations, CNA-24, Center for Numerical Analysis, The University of Texas at Austin, 1971; Numer. Math. (Submitted in revised form.)

6. J. W. SHEldon, "On the spectral norms of several iterative processes," J. Assoc. Comput. Mach., v. 6, 1959, pp. 494-505. MR 23 \#B1647.

7. Richard S. Varga, Matrix Iterative Analysis, Prentice-Hall, Englewood Cliffs, N. J., 1962. MR $28 \# 1725$.

8. E. L. WACHSPress, Iterative Solutions of Elliptic Systems and Applications to the Neutron Diffusion Equations of Reactor Physics, Prentice-Hall, Englewood Cliffs, N. J., 1966 MR 38 \#2965.

9. David M. Young \& Davin R. KIncaid, Norms of the Successive Overrelaxation Method and Related Methods, TNN-94, Computation Center, The University of Texas at Austin, 1969.

10. David M. Young, Convergence Properties of the Symmetric and Unsymmetric Successive Overrelaxation Methods and Related Methods, TNN-96, Computation Center, The University of Texas at Austin, 1969; Math. Comp., v. 24, 1970, pp. 793-807. (Revised version.)

11. Davin M. Young, Itcrative Solution of Large Linear Systems, Academic Press, New York, 1971. 